\title{
Advances in mRNA Silencing and Transgene Expression: a Gateway to Functional Genomics in Schistosomes
}

\author{
Elissaveta B. Tchoubrieva and Bernd H. Kalinna* \\ Centre for Animal Biotechnology, Faculty of Veterinary Science, The University of Melbourne, \\ Parkville, 3010 VIC, Australia
}

\begin{abstract}
The completion of the WHO Schistosoma Genome Project in 2008, although not fully annotated, provides a golden opportunity to actively pursue fundamental research on the parasites genome. This analysis will aid identification of targets for drugs, vaccines and markers for diagnostic tools as well as for studying the biological basis of drug resistance, infectivity and pathology. For the validation of drug and vaccine targets, the genomic sequence data is only of use if functional analyses can be conducted (in the parasite itself). Until recently, gene manipulation approaches had not been seriously addressed. This situation is now changing and rapid advances have been made in gene silencing and transgenesis of schistosomes.
\end{abstract}

\section{Introduction}

Schistosomes are digenetic blood trematodes that cause schistosomiasis in humans by depositing eggs in blood vessels surrounding the bladder or gut of the infected host. In terms of public health, five species of schistosomes are considered most important. They are divided into intestinal schistosomes ( $S$. mansoni, $S$. intercalatum, $S$. japonicum, $S$. mekongi) and urinary schistosomes ( $S$. haematobium) due to the different pathologies they induce, and can be distinguished by the specific snail hosts required for transmission. Schistosomes are spread across Sub-Saharan Africa, South-East Asia and Brazil. The typical transmission site is fresh water ponds (rivers) where the intermediate and the primary hosts come in contact to ensure transmission of the parasite and completion of the life cycle. Schistosomiasis is a major cause of morbidity and mortality and it ranks with malaria and tuberculosis in that regard. It is also amongst the most severe parasitic diseases, threatening millions of people with chronic illness, disfigurement, or death that can result from the parasitic infection and occurs mainly in rural areas of the developing world. The disease is endemic in 76 countries; about 250 million people are globally infected, and almost 800 million are at risk of contracting the infection. The major cause of pathology in schistosomiasis is the schistosome egg, associated with formation of granulomas around trapped eggs in the intestinal wall or in the liver causing fibrosis and hepatosplenomegaly, or eggs can become lodged in the wall of the bladder, resulting in inflammation and blood in the urine. The chronic form of the disease has severe outcomes including ascites (accumulation of fluid in the peritoneal cavity, causing abdominal swelling), liver fibrosis, hepatosplenomegaly, secondary cancers of the bladder and often death. Estimates of the global burden of schistosomiasis range from 1.7 to 4.5 million DALY (disability adjusted life years) (W.H.O., 2002; W.H.O, 2004), with even the higher figure underestimating the true burden as it does not consider chronic long-term disabilities (Hotez et al., 2006). Revised burden estimates for schistosomiasis are in the scale of 3-70 million DALY (King and Dangerfield-

`To whom correspondence may be addressed (bernd.kalinna@unimelb.edu.au). 
Cha, 2008). The current therapeutic approach, chemotherapy with praziquantel, is efficacious against adult worms. But its prolonged use en mass and the fact that praziquantel is ineffective against immature and young adult worms, has already raised concerns about future development of tolerance and drug resistant schistosomes (Utzinger and Keiser, 2004). Vaccines are still not available on the market. It is therefore important to actively pursue fundamental research on parasite genome analysis to identify new targets for drug, vaccine and diagnostics development, and for studying the biological basis of drug resistance, antigenicity, infectivity and pathology. Until very recently, tools for manipulating gene expression in schistosomes have been unavailable, mainly due to the complex developmental life cycle, large genome size and lack of immortalised cell lines.

Schistosomes live as parasites in the liver, gut, lungs or blood vessels of vertebrates and their life cycle involves a mammalian host, where adult worms of the two sexes mate and deposit eggs; a free-living aquatic stage (miracidium), derived from eggs and released into the environment; a molluscan stage; and a second free-living aquatic stage (cercaria). Unlike other trematodes, in the schistosomes the sexes are separate. The male is considerably larger than the female and encloses her within his gynaecophoric canal for the entire adult lives of the worms, where they reproduce sexually. $S$. mansoni has a very large and complex genome (haploid genome is $270 \mathrm{Mb}$ ) organised in 8 pairs of chromosomes (Short, 1983). There are 15-20,000 expressed genes. With the recent completion of the $S$. mansoni sequence genome project (Berriman et al., 2009) and emerging abundance of molecular information, the development of new molecular tools such as RNAi, and the promise of new reliable reagents and techniques for transfection, we have now reached the exciting stage of being able to address important issues in the biology of schistosomes in some detail. This review will focus on the current transgenesis tools available to study the biology and functional genomics of schistosomes and speculate how these can enable us to discover novel genes and biochemical pathways involved in growth, reproduction and survival of the parasite as potential targets for drug and vaccine development.

\section{RNA interference technology (gene silencing)}

The discovery of sequence-specific gene silencing in response to double-stranded RNAs (dsRNA) has had an enormous impact on molecular biology by uncovering an unsuspected layer of gene expression regulation. The process, also known as RNA interference (RNAi) or RNA silencing, involves complementary pairing of dsRNAs with their homologous messenger RNA targets, thereby preventing the expression and leading to subsequent degradation of these mRNAs or interfering with protein translation.

The first evidence that dsRNA could lead to gene silencing came from work in Caenorhabditis elegans published in 1995 by Guo and Kemphues attempting to use antisense RNA to shut down expression of the par-1 gene in order to assess its function and although the injection of the antisense RNA successfully disrupted gene expression, injection of the sense-strand control was equally efficient (Guo and Kemphues, 1995). The explanation for this phenomenon was published a few years later when Fire and colleagues injected a mixture of both sense and antisense strand RNA (dsRNA) into C. elegans, which completely silenced the homologous gene's expression (Fire et al., 1998).

Since then, RNAi technology has been used as a reverse genetics tool in C. elegans, Drosophila and a wide variety of other organisms, including zebrafish, plants, human, mouse and mammalian cell culture, to inhibit gene activity on a post-transcriptional level generating loss-of-function mutants to study gene function or identify and validate novel therapeutic targets (reviewed in Siomi and Siomi, 2009). 
Silencing was found to have high potency and specificity in $C$. elegans since only a few molecules of dsRNA per cell could trigger gene silencing throughout the treated animal (Grishok et al., 2001; Zamore, 2001). The silencing effect usually lasts only for several days however, and although it does appear to be transferred to daughter cells, it eventually diminishes. In some cases it has been shown to have longer-lasting effects as the RNA interference was detected in the first generation progeny of treated C. elegans (Tabara et al., 1998; Grishok and Mello, 2002).

The discovery of RNAi has brought to the scientific community not only a new and exciting biological paradigm, but also an incredibly potent tool for addressing questions of gene function in an easy and high-throughput fashion. Genome-wide RNA interference screens have identified near-complete sets of genes involved in cellular processes. Inducible hairpin RNAi constructs are being applied in studies of complex developmental processes in a tissue-specific manner in Drosophila (Mummery-Widmer et al., 2009).

Recently a number of groups have developed expression vectors to continually express dsRNAs in transiently and stably transfected mammalian cells to overcome the short-term effects of traditional RNAi. Some of these vectors have been engineered to express small hairpin RNAs (shRNAs), which are processed in vivo into dsRNAs (Yu et al., 2002). These vectors contain the shRNA sequence between a promoter and transcription termination site. The transcript folds into a stem-loop structure and the ends of the shRNAs are processed in vivo by an enzyme known as Dicer (ribonuclease III (RNaseIII), converting the shRNAs into 21 nt dsRNA molecules, which in turn initiate RNAi mediated through the RNAinduced silencing complex (RISC).

In schistosomes, the presence of transcripts encoding dicer and RISC-associated proteins (piwi/argonaute orthologues) was relatively recently described by Verjovski-Almeida and colleagues in their report on the transcriptome of S. mansoni (Verjovski-Almeida et al., 2003). This finding indicated that an intact RNAi pathway had evolved in schistosomes. Since then it has been shown that RNAi can be applied in schistosomes and appropriate transformation protocols have been adapted and developed (Table 1).

\section{RNAi in schistosomes}

The first report for successful RNAi in schistosomes, in which cercariae of $S$. mansoni were soaked in dsRNA for 6 days, was published in 2003 (Skelly et al., 2003). The primary objective of this report was to demonstrate the utility of RNAi in schistosomes using the major gut-associated proteinase, cathepsin B (SmCB1or Sm31) as target. Cathepsins are proteolytic enzymes involved in the digestion of hosts haemoglobin during feeding and a principal source of amino acid nutrients for the parasites. Exposure of larval schistosomes to dsRNA, specific to cathepsin B, was demonstrated to inhibit expression of the enzyme. Subsequently, Boyle and colleagues successfully silenced the glucose transporter (SGTP1) and glyceraldehyde-3-phosphate dehydrogenase (GAPDH) genes in sporocysts of $S$. mansoni (Boyle et al., 2003). Exposure of the parasite to SGTP1 dsRNA reduced larval glucose-uptake capacity by $40 \%$, demonstrating a functional phenotype of reduced glucose transport activity. These two publications established that schistosomes were susceptible to RNAi and silencing was strong, specific and long lasting (up to 28 days) in either miracidia or sporocysts.

Within a short time thereafter several laboratories employed RNAi targeting a variety of genes and using different delivery techniques in schistosomes to study gene function. The proteins attracting the most interest were proteolytic enzymes (metallo-, cysteine, and serine proteases) and genes belonging to signalling pathways implicated in adult worm pairing and/ or egg deposition. These two groups of proteins have key functions in schistosomes and 
therefore are potential targets for novel anti-parasite chemotherapy and immunotherapy. The proteases in schistosomes and other parasitic organisms, have been long implicated in pathologies associated with helminth infections and shown to facilitate host invasion (McKerrow and Doenhoff, 1988; McKerrow et al., 1990), parasite feeding (McKerrow and Doenhoff, 1988) and immune evasion (Tamashiro et al., 1987). Schistosomes feed on ingested host blood and the haemoglobin released from erythrocytes is essential for parasite development, growth and reproduction. Blocking proteolysis of host haemoglobin with broad-spectrum protease inhibitors results in profound anti-schistosomal and anti-pathology effects (Bogitsh et al., 1992; Wasilewski et al., 1996), demonstrating the essential role of this pathway in schistosome metabolism. Studies using RNAi approaches alone or in combination with protease specific inhibitors have now been used methodically to study the network of endopeptidases important for the intestinal protein digestion in $S$. mansoni (Correnti et al., 2005; Delcroix et al., 2006; Morales et al., 2008). It has been shown that initial degradation of host blood proteins is ordered, occasionally redundant, and substratespecific, while the RNAi mediated silencing effect was strong (up to 80\%), target specific and prolonged. The schistosomes treated with dsRNA to SmCB1 were viable, with typical intestinal haematin pigmentation (the result of haemoglobin digestion) and exhibited a significant growth retardation phenotype (Figure 1), indicating the essential nature of this complex protease network which is highly conserved throughout invertebrate evolution (Correnti et al., 2005). In experiments targeting another endopeptidase, cathepsin D, by electroporation with dsRNA it was shown that haematin was apparently not deposited in the gut of schistosomules as it appeared red in colour, indicating the presence of intact rather than digested host haemoglobin (Morales et al., 2008). These schistosomules did not survive to maturity after transfer into mice confirming the essential function this enzyme has in parasite nutrition and its potential as target for novel anti-schistosomal interventions.

Another schistosome protease - the asparaginyl endopeptidase SmAE (also known as Sm32, or legumain), has been proposed to proteolytically convert the inactive precursor of SmCB1 into its mature catalytic form in vitro (Dalton and Brindley, 1996; Caffrey et al., 2004).

Although a substantial and specific suppression (>90\%) of SmAE transcripts was achieved by RNAi and SmAE enzyme activity could not be detected, it was shown that SmCB1 was fully processed and active. This finding indicated that SmAE was not essential in SmCB1 activation in vivo (Krautz-Peterson and Skelly, 2008).

The protocols for effective gene silencing by RNAi targeting cathepsin B in life stages of $S$. mansoni have been summarised by Krautz-Peterson and co-workers (Krautz-Peterson et al., 2007). In brief, the conclusions were that electroporation was more effective in delivering dsRNA in schistosomula compared to soaking and that both small interfering RNAs (siRNAs) ( $21 \mathrm{bp}$ ) and long dsRNA (>405 bp) demonstrated similar silencing efficiency. Furthermore, neither the persistence of the dsRNA in culture medium, nor transformation of cercariae to schistosomula adversely affected RNAi susceptibility and silencing.

Interestingly, complete suppression of the cathepsin B gene was never achieved regardless of the dsRNA dose, possibly due to difficulties to achieve gene silencing uniformly in a mixed population of cells in a living worm.

Research efforts in a number of laboratories are aimed at understanding the role of signal transduction pathways and their role in parasite interaction with its host environment and amongst themselves. For example, the TGF- $\beta$ signalling pathway is essential for schistosome embryogenesis and has implications for the development of novel therapeutic strategies and prevention of the disease (reviewed in Ting-An and Hong-Xiang, 2009).

Schistosomes express a TGF- $\beta$ type II receptor (SmT $\beta$ RII) and are exceptional amongst the trematodes in the way that they have evolved separate sexes and the sexual development of the female requires constant contact with the male. This unique phenomenon is associated 
with pathogenesis since eggs are the triggering factor, not the adult worms themselves (reviewed in Hoffmann, 2004). Thus, blocking components of the parasite TGF- $\beta$ signalling pathway by RNAi would likely abolish worm pairing and egg production, rendering them potential targets for novel intervention strategies development towards transmission and disease control (Osman et al., 2006; Freitas et al., 2007).

RNAi was also applied in $S$. japonicum to investigate the role of the gynaecophoral canal protein SjGCP during the pairing process (Cheng et al., 2005). The pairing of a male worm with a female worm residing in the gynaecophoral canal of the male plays a critical role in the development of the female parasite. Because the male-specific SjGCP is found in significant quantities in the adult female worm after pairing, it could play an important role in parasite pairing. Dose-dependent inhibition with up to $75 \%$ suppression was observed on the SjGCP transcript level in schistosomules 7 days after treatment with siRNA. In further studies, the effect of siRNA duplexes targeting the SjGCP gene was evaluated in vitro as well as in mice infected with $S$. japonicum in vivo (Cheng et al., 2009). Two out of three siRNAs employed gave rise to significant suppression of SjGCP transcript levels, reduction of protein amount, and totally abolished the parasite pairing. Evaluation of the pairing inhibitory effect in vivo, in mice infected with $S$. japonicum and treated with siRNA, revealed significant inhibition of early parasite pairing and reduced parasite burden, a demonstration of the important role of SjGCP in pairing and subsequent development of $S$. japonicum.

Vector-mediated gene silencing by siRNAs derived from shRNA expressed by mammalian Pol III promoter H1 is also applicable in schistosomes (Zhao et al., 2008). Schistosomula were electroporated with a Mago nashi shRNA expression vector. The shRNA expressed from the mammalian Pol III promoter H1 specifically reduced the levels of Mago nashi mRNA and proteins in $S$. japonicum accompanied by pronounced phenotypic changes in the testicular lobes.

RNAi in schistosomes has provided means for functional analysis of genes such as a CD36like class B scavenger receptor (SRB) which is potentially involved in some aspect of larval growth and development (Dinguirard and Yoshino, 2006) and an $S$. mansoni alkaline phosphatase (SmAP) (Ndegwa et al., 2007). The use of RNAi technology further suggested that the proteasome may be downregulated during the early stages of schistosomula development and is subsequently upregulated as the parasite matures to the adult stage (Nabhan et al., 2007). Kumagai and colleagues uncovered the function of Peroxiredoxin-1 (Prx-1) in $S$. japonicum as a scavenger against hydrogen peroxide showing its potential as novel target for drug and vaccine development for schistosomiasis (Kumagai et al., 2009). Both genetic and biochemical approaches were employed in the discovery and validation of potential drug target meeting all the major criteria for anti-schistosomal chemotherapy development. Silencing of the thioredoxin glutathione reductase (TGR) by RNAi led to rapid parasite death within 4 days of treatment, proving its essential role in the survival of $S$. mansoni. Use of specific and efficient inhibitors of TGR reduced the worm burden by $\sim 60 \%$, suggesting the importance of TGR for parasite survival and its potential as key target for therapy with those compounds (Kuntz et al., 2007).

The first successful in vivo demonstration and evaluation of the therapeutic application of RNAi against schistosomiasis in a chronic infection model has been recently published by Pereira and colleagues. Small interfering RNAs were produced against the hypoxanthine guanine phosphoribosyl transferase (HGPRTase) gene in S. mansoni and intravenously injected into infected mice. As a result the total number of parasites was reduced by approximately $27 \%$. RT-PCR analysis showed a significant reduction in parasite target mRNA but not in the hosts homologue. The survival rate of treated mice was not affected by 
the dose of siRNAs. Further optimization in molecule delivery and siRNA dose could be expected to have a more pronounced effect on the parasite and possibly lead to its complete elimination (Pereira et al., 2008).

\section{Transgenesis of schistosomes}

Since completion of the Schistosoma mansoni genome sequencing project in 2008, the challenge we now face is how to determine the function of unknown genes and pathways involved in host immune evasion, growth, reproduction and survival, all of which potentially represent novel more effective targets for drug and vaccine development. The development of tools and methods for gene manipulation in schistosomes has been slow due to their complex developmental life cycle, large size genome and absence of immortalised cell lines. Several approaches for the introduction of transgenes (transgenesis) in the form of reporter gene RNA or plasmid based cDNA into schistosomes have been adapted and significant advances have been made (Table 2). A number of approaches are theoretically feasible to introduce reporter transgenes into schistosomes. These include microinjection, electroporation, lipofection-type approaches, biolistics (particle bombardment), or the use of infectious vectors such as retrovirus or mobile genetic elements.

In pioneering studies transgenes in the form of mRNA or plasmids were introduced into the parasites by particle bombardment (Davis et al., 1999; Wippersteg et al., 2002a; Heyers et al., 2003). It is now a decade ago that in a landmark paper Davis and colleagues described the delivery of luciferase by mRNA or encoded on a DNA plasmid into adult schistosomes by particle bombardment (Davis et al., 1999). The DNA plasmid contained the $S$. mansoni SL RNA gene fused upstream of the luciferase ORF followed by an $S$. mansoni enolase UTR and polyadenylation signal. With both mRNA and plasmid-encoded luciferase the authors detected reporter protein expression by luminometry. Luciferase was present and expressed 24 hours after particle bombardment.

After this initial paper a number of reports were published in short succession, also employing particle bombardment (Wippersteg et al., 2002a; Wippersteg et al., 2002b; Heyers et al., 2003; Rossi et al., 2003; Wippersteg et al., 2003). Wippersteg et al. constructed a plasmid expressing the Green Fluorescent Protein (GFP) reporter gene under the control of the $S$. mansoni HSP70 promoter and terminator. This plasmid was introduced into sporocysts and adults and expression of GFP could be shown after heat shock induction by confocal microscopy 3 days after transfection. Fluorescence was mainly visible on the surface of adult worms and inside sporocysts. The authors also employed RT-PCR to detect GFP transcripts and Western Blotting to identify the GFP protein (Wippersteg et al., 2002a).

Expanding on this report Wippersteg and co-workers in the same year published further experiments where the SmHSP70 promoter and terminator elements in the plasmid used for transfection were exchanged for cis-acting elements of the S. mansoni ER60 (SmER60) gene (Wippersteg et al., 2002b). SmER60 encodes a cysteine protease which in earlier studies had been localised to the endoplasmic reticulum in excretory tissues in adult parasites (Finken-Eigen and Kunz, 1997). After bombardment of sporocysts the observed expression of GFP was tissue-specific, and the localisation of ER60 in the excretory/ secretory (ES) system of the larval parasites suggested that ER60 might have a role in penetration and migration of miracidia in the intermediate snail host. In an additional follow-up report the same authors verified this tissue specific expression of the ER60 protease by employing Texas Red-labelled BSA, which accumulates in the ES system, together with biolistic transformation. The ER60-GFP and the Texas Red-BSA co-localised in the same compartments (Wippersteg et al., 2003). 
The same approach to co-localise Texas Red-BSA to the ES system was used by Rossi et al. to study $S$. mansoni calcineurin A and its expression in the ES system (Rossi et al., 2003). A plasmid containing 5' - and 3'-elements of the calcineurin A gene, driving the expression of GFP was constructed and biolistically transferred into sporocysts and male adult worms. Similar to the results discussed above, fluorescent signals for GFP and Texas Red colocalised in the ES system of the parasite.

In our laboratory we introduced plasmid DNA by particle bombardment into miracidia, sporocysts and adults of $S$. mansoni (Heyers et al., 2003). In these studies the Enhanced Green Fluorescent Protein (EGFP) was used in a plasmid similar to that used be Wippersteg described above. In our studies we particularly focused on the miracidial life cycle stage, because this larval stage offers the unique opportunity to introduce transgenes into the germline and additionally to reintroduce transgenic organisms into the parasite life cycle. Bombarded miracidia were able to infect $B$. glabrata snail and two weeks after infection gold particles could clearly be identified within the developing sporocysts in paraffinsections of infected snail tissues. Interestingly, these gold particles were located close to the nuclei of germ ball cells (Figure 2). Transfection of these totipotent cells could, theoretically, lead to the transformation of the germ line and the derivation of transgenic schistosomes. Reporter gene activity could also be determined at 10 days post-infection by RT-PCR. These findings indicated that it is possible to return transgenic miracidia to the parasite life cycle, a crucial step for the establishment of a transgenesis system for schistosomes.

The most recent publication describing the transfection of schistosomes using biolistic methods reports the tissue-specific expression of GFP driven by the promoters of two of $S$. mansoni protease genes, cathepsin L1 and cathepsin B2 (Wippersteg et al., 2005). As predicted from earlier reports (Bogitsh et al., 2001), the $S$. mansoni cathepsin L1 promoter drove GFP expression throughout the gut whereas transformation with the SmCB2 (Caffrey et al., 2002) construct resulted in GFP fluorescence localised in the tegument.

More recently the efficacy of electroporation for the introduction of plasmid-based DNA constructs was tested in S. japonicum and S. mansoni (Yuan et al., 2005; Correnti et al., 2007). Yuan et al. using the commercial plasmid pEGFP-C1 (Clontech) were able to show that the CMV promoter could drive EGFP expression in primary cells cultures of $S$. japonicum. Additionally, the plasmids were introduced into schistosomula and adult worms by electroporation and EGFP expression was demonstrated using RT-PCR, Western Blotting and confocal microscopy, which revealed EGFP fluorescence along the tegumental surface of the worms (Yuan et al., 2005). In their report Correnti and colleagues describe the use of electroporation for the delivery of plasmid DNA into larval $S$. mansoni schistosomes, combined with a preliminary analysis of the $S$. mansoni Actin 1.1 (SmAct1.1) promoter sequence (Correnti et al., 2007). Expression of luciferase driven by the SmAct 1.1 gene promoter was transient. The authors suggest that the loss of expression over time was probably not due to loss of plasmid, because transfected parasites that were no longer expressing the luciferase remained PCR-positive for luciferase DNA for 8 weeks following electroporation. This finding is similar to that reported by Yuan et al. (Yuan et al., 2005). These results also indicated that the electroporation protocol described was either insufficient to deliver the transgene to the germline or that the transgene was not integrated at high frequency to be able to be detected in transgenic F1 parasites.

Although these reports clearly showed advances in the development of molecular tools to characterise schistosome genes, it is unlikely that transfection with plasmid based transgenes will result in integration into the genome. Integration into the genome would represent a key milestone for the study of long-term transgene expression, investigation of schistosome gene 
function, development of transgenic schistosomes, and insertional mutagenesis approaches. This might be achievable with gene therapy-type approaches utilising retroviruses, lentiviruses, retrotransposons, or transposons which enhance the likelihood of development of heritable, transgenic lines of schistosomes. This is particularly likely if germ-line cells can be targeted for transduction. Additionally, transposons or retroviruses can carry gene cassettes for production of short interfering RNAs and thereby combine a powerful knockdown technology with an efficient delivery system offering the possibility for heritable RNAi targeting specific host cell genes (Brown et al., 2003; Paddison et al., 2004).

Together with colleagues we have recently reported approaches using retroviruses and transposons to transduce schistosomes (Kines et al., 2006; Morales et al., 2007). In Kines et al. we produced replication incompetent Moloney Murine Leukaemia Virus (MMLV) virions pseudotyped with Vesicular Stomatitis Virus Glycoprotein (VSVG) and carrying a luciferase reporter gene. After exposure of schistosomes to virions harvested from producer cells cultures, immunofluorescence studies indicated that the VSVG envelope interacted with the schistosome surface and that the retroviral capsid and RNA genome were released within the surface cells (Figure 3). We were also able to show the presence of proviral forms of the retrovirus within the schistosome genome by Southern hybridization analysis, while transcription from the transgenes was indicated by the presence of transcripts encoding neomycin phosphotransferase and luciferase. These results strongly suggested integration of the retroviral transgenes into schistosome chromosomes (Kines et al., 2006).

We have also used the transposon piggyBac to accomplish transformation of $S$. mansoni (Morales et al., 2007). A piggyBac donor plasmid containing the luciferase-coding sequence under the control of the SmAct1.1 promoter (Correnti et al., 2007) was constructed and transfected into schistosome by electroporation of larval stages together with mRNAs encoding the piggyBac transposase. The activity of piggyBac was determined by plasmid excision assays, and the recovery of excised plasmids from tissues of transformed schistosomules in these assays indicated that piggyBac was active in the worm. Southern blot hybridisation analysis of genomic DNAs from populations of schistosomules transformed with donor constructs and helper transposase-mRNA detected numerous variable length luciferase-positive signals. These findings further indicated piggyBac transposon insertions into the schistosome chromosomes. The integration sites were analysed by a PCR technique. Numerous piggyBac integrations were detected and, after cloning, the fragments sequenced ranged in size from $\sim 1.5 \mathrm{~kb}$ to $4 \mathrm{~kb}$. Sequence analysis indicated that integration of piggyBac took place at numerous loci in the schistosome genome at target TTAA sites.

Current studies in our laboratory are aimed at vector mediated RNAi of the schistosomal cathepsin B1 protease (SmCatB1), using this viral system described above (Kines et al., 2006). We have designed viral vectors to express targeted dsRNA, thus coupling a powerful delivery vehicle with a potent mechanism to specifically silence a gene of interest. We have targeted SmCatB1 because RNAi knockdown of this protein delivers a visible phenotype (Correnti et al., 2005). Preliminary studies have shown that the newly developed viral vector for the production of virions carrying a dsRNA hairpin loop specific for SmCatB1 resulted in $80 \%$ reduction in transcript levels of this protease $72 \mathrm{~h}$ after exposure to the virus and an effective silencing of the cathepsin B enzyme (Tchoubrieva et al., unpublished).

\section{Concluding remarks and outlook}

The genome of S. mansoni (Berriman et al., 2009; Zerlotini et al., 2009) marks the first step toward a better understanding of this destructive, blood-feeding parasite of humans. Genomic sequence data provide a rich resource for gene expression profiling using 
microchip gene array analysis, and for gene deletion studies and/or gene silencing. Thus, the availability of the complete genome sequence of $S$. mansoni and emerging technologies now provide a golden opportunity to explore the function of schistosome genes and gene products. Driven by this rapidly growing information on genome sequences the progress in schistosome RNAi and transgenesis over the last few years has been remarkable and existing technologies have been rapidly adapted for use with these parasites.

An exciting approach which shows promise for conducting effective functional analyses in schistosomes is the development of promoter trap or polyA trap vectors. Gene trapping is a high-throughput approach used to introduce insertional mutations across the genome. Gene traps are able to simultaneously inactivate and report the expression of the trapped gene at the insertion site, and provide a DNA tag for the rapid identification of the disrupted gene (Zambrowicz et al., 1998; Stanford et al., 2001). Gene trapping has been used in mouse, zebrafish and Drosophila genomics and has proven to be a powerful method to identify functional genes (Wurst et al., 1995; Hicks et al., 1997; Lukacsovich and Yamamoto, 2001; Jao et al., 2008). We are now pursuing the gene trapping approach as the next step towards functional genomics of schistosomes to ultimately establish a correlation between the physical and genetic maps of the schistosome genome.

\section{Acknowledgments}

We thank Anna Walduck for helpful discussions and critical review of the manuscript. Support from the National Health \& Medical Research Council of Australia (454422) and the US National Institutes of Health (RO1AI072773) is gratefully acknowledged.

\section{Abbreviations}

$\begin{array}{ll}\text { WHO } & \text { World Health Organization } \\ \text { DALY } & \text { Disability Adjusted Life Years } \\ \text { dSRNA } & \text { double stranded RNA } \\ \text { RNAi } & \text { RNA interference } \\ \text { RISC } & \text { RNA-Induced Silencing Complex } \\ \text { siRNA } & \text { small interfering RNA } \\ \text { MMLV } & \text { Moloney Murine Leukaemia Virus } \\ \text { VSVG } & \text { Vesicular Stomatitis Virus Glycoprotein } \\ \text { HSP70 } & \text { Heat Shock Protein 70 } \\ \text { RT-PCR } & \text { Real Time Polymerase Chain Reaction } \\ \text { CMV } & \text { Cytomegalovirus } \\ \text { ORF } & \text { Open Reading Frame } \\ \text { UTR } & \text { Untranslated Region }\end{array}$

\section{References}

Berriman M, Haas BJ, Loverde PT, Wilson RA, Dillon GP, Cerqueira GC, Mashiyama ST, AlLazikani B, Andrade LF, Ashton PD, Aslett MA, Bartholomeu DC, Blandin G, Caffrey CR, Coghlan A, Coulson R, Day TA, Delcher A, Demarco R, Djikeng A, Eyre T, Gamble JA, Ghedin E, Gu Y, Hertz-Fowler C, Hirai H, Hirai Y, Houston R, Ivens A, Johnston DA, Lacerda D, Macedo CD, Mcveigh P, Ning Z, Oliveira G, Overington JP, Parkhill J, Pertea M, Pierce RJ, Protasio AV, Quail MA, Rajandream MA, Rogers J, Sajid M, Salzberg SL, Stanke M, Tivey AR, White O, 
Williams DL, Wortman J, Wu W, Zamanian M, Zerlotini A, Fraser-Liggett CM, Barrell BG, ElSayed NM. The genome of the blood fluke Schistosoma mansoni. Nature. 2009; 460:352-358. [PubMed: 19606141]

Bogitsh BJ, Dalton JP, Brady CP, Brindley PJ. Gut-associated immunolocalization of the Schistosoma mansoni cysteine proteases, SmCL1 and SmCL2. J Parasitol. 2001; 87:237-241. [PubMed: 11318550]

Bogitsh BJ, Kirschner KF, Rotmans JP. Schistosoma japonicum: immunoinhibitory studies on hemoglobin digestion using heterologous antiserum to bovine cathepsin D. J Parasitol. 1992; 78:454-459. [PubMed: 1597788]

Boyle JP, Wu XJ, Shoemaker CB, Yoshino TP. Using RNA interference to manipulate endogenous gene expression in Schistosoma mansoni sporocysts. Mol Biochem Parasitol. 2003; 128:205-215. [PubMed: 12742587]

Brown AE, Bugeon L, Crisanti A, Catteruccia F. Stable and heritable gene silencing in the malaria vector Anopheles stephensi. Nucleic Acids Res. 2003; 31:e85. [PubMed: 12888537]

Caffrey CR, McKerrow JH, Salter JP, Sajid M. Blood 'n' guts: an update on schistosome digestive peptidases. Trends Parasitol. 2004; 20:241-248. [PubMed: 15105025]

Caffrey CR, Salter JP, Lucas KD, Khiem D, Hsieh I, Lim KC, Ruppel A, McKerrow JH, Sajid M. SmCB2, a novel tegumental cathepsin B from adult Schistosoma mansoni. Mol Biochem Parasitol. 2002; 121:49-61. [PubMed: 11985862]

Cheng G, Fu Z, Lin J, Shi Y, Zhou Y, Jin Y, Cai Y. In vitro and in vivo evaluation of small interference RNA-mediated gynaecophoral canal protein silencing in Schistosoma japonicum. J Gene Med. 2009; 11:412-421. [PubMed: 19288459]

Cheng GF, Lin JJ, Shi Y, Jin YX, Fu ZQ, Jin YM, Zhou YC, Cai YM. Dose-dependent inhibition of gynecophoral canal protein gene expression in vitro in the schistosome (Schistosoma japonicum) by RNA interference. Acta Biochim Biophys Sin (Shanghai). 2005; 37:386-390. [PubMed: 15944753]

Correnti JM, Brindley PJ, Pearce EJ. Long-term suppression of cathepsin B levels by RNA interference retards schistosome growth. Mol Biochem Parasitol. 2005; 143:209-215. [PubMed: 16076506]

Correnti JM, Jung E, Freitas TC, Pearce EJ. Transfection of Schistosoma mansoni by electroporation and the description of a new promoter sequence for transgene expression. Int J Parasitol. 2007; 37:1107-1115. [PubMed: 17482194]

Dalton JP, Brindley PJ. Schistosome asparaginyl endopeptidase SM32 in hemoglobin digestion. Parasitol Today. 1996; 12:125. [PubMed: 15275249]

Davis RE, Parra A, LoVerde PT, Ribeiro E, Glorioso G, Hodgson S. Transient expression of DNA and RNA in parasitic helminths by using particle bombardment. Proc Natl Acad Sci U S A. 1999; 96:8687-8692. [PubMed: 10411936]

Delcroix M, Sajid M, Caffrey CR, Lim KC, Dvorak J, Hsieh I, Bahgat M, Dissous C, McKerrow JH. A multienzyme network functions in intestinal protein digestion by a platyhelminth parasite. J Biol Chem. 2006; 281:39316-39329. [PubMed: 17028179]

Dinguirard N, Yoshino TP. Potential role of a CD36-like class B scavenger receptor in the binding of modified low-density lipoprotein (acLDL) to the tegumental surface of Schistosoma mansoni sporocysts. Mol Biochem Parasitol. 2006; 146:219-230. [PubMed: 16427708]

Finken-Eigen M, Kunz W. Schistosoma mansoni: gene structure and localization of a homologue to cysteine protease ER 60. Exp Parasitol. 1997; 86:1-7. [PubMed: 9149235]

Fire A, Xu S, Montgomery MK, Kostas SA, Driver SE, Mello CC. Potent and specific genetic interference by double-stranded RNA in Caenorhabditis elegans. Nature. 1998; 391:806-811. [PubMed: 9486653]

Freitas TC, Jung E, Pearce EJ. TGF-beta signaling controls embryo development in the parasitic flatworm Schistosoma mansoni. PLoS Pathog. 2007; 3:e52. [PubMed: 17411340]

Grishok A, Mello CC. RNAi (Nematodes: Caenorhabditis elegans). Adv Genet. 2002; 46:339-360. [PubMed: 11931230]

Grishok A, Pasquinelli AE, Conte D, Li N, Parrish S, Ha I, Baillie DL, Fire A, Ruvkun G, Mello CC. Genes and mechanisms related to RNA interference regulate expression of the small temporal RNAs that control C. elegans developmental timing. Cell. 2001; 106:23-34. [PubMed: 11461699] 
Guo S, Kemphues KJ. par-1, a gene required for establishing polarity in $C$. elegans embryos, encodes a putative Ser/Thr kinase that is asymmetrically distributed. Cell. 1995; 81:611-620. [PubMed: 7758115]

Heyers O, Walduck AK, Brindley PJ, Bleiss W, Lucius R, Dorbic T, Wittig B, Kalinna BH. Schistosoma mansoni miracidia transformed by particle bombardment infect Biomphalaria glabrata snails and develop into transgenic sporocysts. Exp Parasitol. 2003; 105:174-178. [PubMed: 14969695]

Hicks GG, Shi EG, Li XM, Li CH, Pawlak M, Ruley HE. Functional genomics in mice by tagged sequence mutagenesis. Nat Genet. 1997; 16:338-344. [PubMed: 9241269]

Hoffmann KF. An historical and genomic view of schistosome conjugal biology with emphasis on sexspecific gene expression. Parasitology. 2004; 128(Suppl 1):S11-S22. [PubMed: 16454894]

Hotez PJ, Molyneux DH, Fenwick A, Ottesen E, Ehrlich Sachs S, Sachs JD. Incorporating a rapidimpact package for neglected tropical diseases with programs for HIV/AIDS, tuberculosis, and malaria. PLoS Med. 2006; 3:e102. [PubMed: 16435908]

Jao LE, Maddison L, Chen W, Burgess SM. Using retroviruses as a mutagenesis tool to explore the zebrafish genome. Brief Funct Genomic Proteomic. 2008; 7:427-443. [PubMed: 18977782]

Kines KJ, Mann VH, Morales ME, Shelby BD, Kalinna BH, Gobert GN, Chirgwin SR, Brindley PJ. Transduction of Schistosoma mansoni by vesicular stomatitis virus glycoprotein-pseudotyped Moloney murine leukemia retrovirus. Exp Parasitol. 2006; 112:209-220. [PubMed: 16530185]

Kines KJ, Morales ME, Mann VH, Gobert GN, Brindley PJ. Integration of reporter transgenes into Schistosoma mansoni chromosomes mediated by pseudotyped murine leukemia virus. FASEB J. 2008; 22:2936-2948. [PubMed: 18403630]

King CH, Dangerfield-Cha M. The unacknowledged impact of chronic schistosomiasis. Chronic Illn. 2008; 4:65-79. [PubMed: 18322031]

Krautz-Peterson G, Radwanska M, Ndegwa D, Shoemaker CB, Skelly PJ. Optimizing gene suppression in schistosomes using RNA interference. Mol Biochem Parasitol. 2007; 153:194-202. [PubMed: 17420062]

Krautz-Peterson G, Skelly PJ. Schistosome asparaginyl endopeptidase (legumain) is not essential for cathepsin B1 activation in vivo. Mol Biochem Parasitol. 2008; 159:54-58. [PubMed: 18280591]

Kumagai T, Osada Y, Ohta N, Kanazawa T. Peroxiredoxin-1 from Schistosoma japonicum functions as a scavenger against hydrogen peroxide but not nitric oxide. Mol Biochem Parasitol. 2009; 164:26-31. [PubMed: 19041905]

Kuntz AN, Davioud-Charvet E, Sayed AA, Califf LL, Dessolin J, Arner ES, Williams DL. Thioredoxin glutathione reductase from Schistosoma mansoni: an essential parasite enzyme and a key drug target. PLoS Med. 2007; 4:e206. [PubMed: 17579510]

Lukacsovich T, Yamamoto D. Trap a gene and find out its function: toward functional genomics in Drosophila. J Neurogenet. 2001; 15:147-168. [PubMed: 12092900]

McKerrow JH, Brindley P, Brown M, Gam AA, Staunton C, Neva FA. Strongyloides stercoralis: identification of a protease that facilitates penetration of skin by the infective larvae. Exp Parasitol. 1990; 70:134-143. [PubMed: 2137091]

McKerrow JH, Doenhoff MJ. Schistosome proteases. Parasitol Today. 1988; 4:334-340. [PubMed: 15463025]

Morales ME, Mann VH, Kines KJ, Gobert GN, Fraser MJ Jr, Kalinna BH, Correnti JM, Pearce EJ, Brindley PJ. piggyBac transposon mediated transgenesis of the human blood fluke, Schistosoma mansoni. FASEB J. 2007; 21:3479-3489. [PubMed: 17586730]

Morales ME, Rinaldi G, Gobert GN, Kines KJ, Tort JF, Brindley PJ. RNA interference of Schistosoma mansoni cathepsin D, the apical enzyme of the hemoglobin proteolysis cascade. Mol Biochem Parasitol. 2008; 157:160-168. [PubMed: 18067980]

Mummery-Widmer JL, Yamazaki M, Stoeger T, Novatchkova M, Bhalerao S, Chen D, Dietzl G, Dickson BJ, Knoblich JA. Genome-wide analysis of Notch signalling in Drosophila by transgenic RNAi. Nature. 2009; 458:987-992. [PubMed: 19363474]

Nabhan JF, El-Shehabi F, Patocka N, Ribeiro P. The 26S proteasome in Schistosoma mansoni: bioinformatics analysis, developmental expression, and RNA interference (RNAi) studies. Exp Parasitol. 2007; 117:337-347. [PubMed: 17892869] 
Ndegwa D, Krautz-Peterson G, Skelly PJ. Protocols for gene silencing in schistosomes. Exp Parasitol. 2007; 117:284-291. [PubMed: 17870072]

Osman A, Niles EG, Verjovski-Almeida S, LoVerde PT. Schistosoma mansoni TGF-beta receptor II: role in host ligand-induced regulation of a schistosome target gene. PLoS Pathog. 2006; 2:e54. [PubMed: 16789838]

Paddison PJ, Caudy AA, Sachidanandam R, Hannon GJ. Short hairpin activated gene silencing in mammalian cells. Methods Mol Biol. 2004; 265:85-100. [PubMed: 15103070]

Pereira TC, Pascoal VD, Marchesini RB, Maia IG, Magalhaes LA, Zanotti-Magalhaes EM, LopesCendes I. Schistosoma mansoni: evaluation of an RNAi-based treatment targeting HGPRTase gene. Exp Parasitol. 2008; 118:619-623. [PubMed: 18237732]

Rossi A, Wippersteg V, Klinkert MQ, Grevelding CG. Cloning of 5' and 3' flanking regions of the Schistosoma mansoni calcineurin A gene and their characterization in transiently transformed parasites. Mol Biochem Parasitol. 2003; 130:133-138. [PubMed: 12946850]

Short RB. Presidential address: Sex and the single schistosome. J Parasitol. 1983; 69:3-22. [PubMed: 6338197]

Siomi H, Siomi MC. On the road to reading the RNA-interference code. Nature. 2009; 457:396-404. [PubMed: 19158785]

Skelly PJ, Da'dara A, Harn DA. Suppression of cathepsin B expression in Schistosoma mansoni by RNA interference. Int J Parasitol. 2003; 33:363-369. [PubMed: 12705930]

Stanford WL, Cohn JB, Cordes SP. Gene-trap mutagenesis: past, present and beyond. Nat Rev Genet. 2001; 2:756-768. [PubMed: 11584292]

Tabara H, Grishok A, Mello CC. RNAi in C. elegans: soaking in the genome sequence. Science. 1998; 282:430-431. [PubMed: 9841401]

Tamashiro WK, Rao M, Scott AL. Proteolytic cleavage of IgG and other protein substrates by Dirofilaria immitis microfilarial enzymes. J Parasitol. 1987; 73:149-154. [PubMed: 3553516]

Ting-An W, Hong-Xiang Z. PTK-pathways and TGF-beta signaling pathways in schistosomes. J Basic Microbiol. 2009; 49:25-31. [PubMed: 19253328]

Utzinger J, Keiser J. Schistosomiasis and soil-transmitted helminthiasis: common drugs for treatment and control. Expert Opin Pharmacother. 2004; 5:263-285. [PubMed: 14996624]

Verjovski-Almeida S, DeMarco R, Martins EA, Guimaraes PE, Ojopi EP, Paquola AC, Piazza JP, Nishiyama MY Jr, Kitajima JP, Adamson RE, Ashton PD, Bonaldo MF, Coulson PS, Dillon GP, Farias LP, Gregorio SP, Ho PL, Leite RA, Malaquias LC, Marques RC, Miyasato PA, Nascimento AL, Ohlweiler FP, Reis EM, Ribeiro MA, Sa RG, Stukart GC, Soares MB, Gargioni C, Kawano T, Rodrigues V, Madeira AM, Wilson RA, Menck CF, Setubal JC, Leite LC, Dias-Neto E.

Transcriptome analysis of the acoelomate human parasite Schistosoma mansoni. Nat Genet. 2003; 35:148-157. [PubMed: 12973350]

W.H.O. The global burden of disease: 2004 update. 2004.

W.H.O. Prevention and control of schistosomiasis and soil-transmitted helminthiasis: report of a WHO expert committee, Geneva 2002. WHO Technical Report Series. 2002; 912:1-57.

Wasilewski MM, Lim KC, Phillips J, McKerrow JH. Cysteine protease inhibitors block schistosome hemoglobin degradation in vitro and decrease worm burden and egg production in vivo. Mol Biochem Parasitol. 1996; 81:179-189. [PubMed: 8898333]

Wippersteg V, Kapp K, Kunz W, Grevelding CG. Characterisation of the cysteine protease ER60 in transgenic Schistosoma mansoni larvae. Int J Parasitol. 2002b; 32:1219-1224. [PubMed: 12204221]

Wippersteg V, Kapp K, Kunz W, Jackstadt WP, Zahner H, Grevelding CG. HSP70-controlled GFP expression in transiently transformed schistosomes. Mol Biochem Parasitol. 2002a; 120:141-150. [PubMed: 11849713]

Wippersteg V, Ribeiro F, Liedtke S, Kusel JR, Grevelding CG. The uptake of Texas Red-BSA in the excretory system of schistosomes and its colocalisation with ER60 promoter-induced GFP in transiently transformed adult males. Int J Parasitol. 2003; 33:1139-1143. [PubMed: 13678630]

Wippersteg V, Sajid M, Walshe D, Khiem D, Salter JP, McKerrow JH, Grevelding CG, Caffrey CR. Biolistic transformation of Schistosoma mansoni with 5' flanking regions of two peptidase genes promotes tissue-specific expression. Int J Parasitol. 2005; 35:583-589. [PubMed: 15862572] 
Wurst W, Rossant J, Prideaux V, Kownacka M, Joyner A, Hill DP, Guillemot F, Gasca S, Cado D, Auerbach A, et al. A large-scale gene-trap screen for insertional mutations in developmentally regulated genes in mice. Genetics. 1995; 139:889-899. [PubMed: 7713439]

Yu JY, DeRuiter SL, Turner DL. RNA interference by expression of short-interfering RNAs and hairpin RNAs in mammalian cells. Proc Natl Acad Sci U S A. 2002; 99:6047-6052. [PubMed: 11972060]

Yuan XS, Shen JL, Wang XL, Wu XS, Liu DP, Dong HF, Jiang MS. Schistosoma japonicum: a method for transformation by electroporation. Exp Parasitol. 2005; 111:244-249. [PubMed: 16212956]

Zambrowicz BP, Friedrich GA, Buxton EC, Lilleberg SL, Person C, Sands AT. Disruption and sequence identification of 2,000 genes in mouse embryonic stem cells. Nature. 1998; 392:608611. [PubMed: 9560157]

Zamore PD. RNA interference: listening to the sound of silence. Nat Struct Biol. 2001; 8:746-750. [PubMed: 11524674]

Zerlotini A, Heiges M, Wang H, Moraes RL, Dominitini AJ, Ruiz JC, Kissinger JC, Oliveira G. SchistoDB: a Schistosoma mansoni genome resource. Nucleic Acids Res. 2009; 37:D579-D582. [PubMed: 18842636]

Zhao ZR, Lei L, Liu M, Zhu SC, Ren CP, Wang XN, Shen JJ. Schistosoma japonicum: inhibition of Mago nashi gene expression by shRNAmediated RNA interference. Exp Parasitol. 2008; 119:379_ 384. [PubMed: 18466902] 

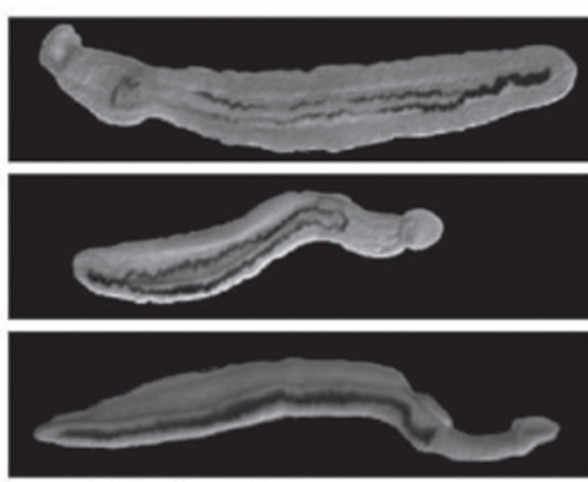

(A) L-dsRNA Treated
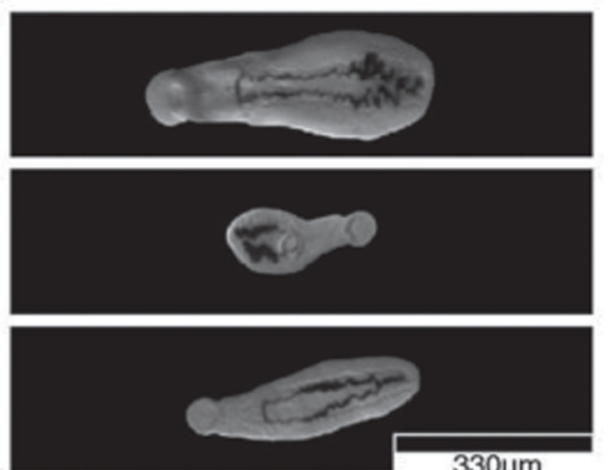

SmCB1-dsRNA Treated

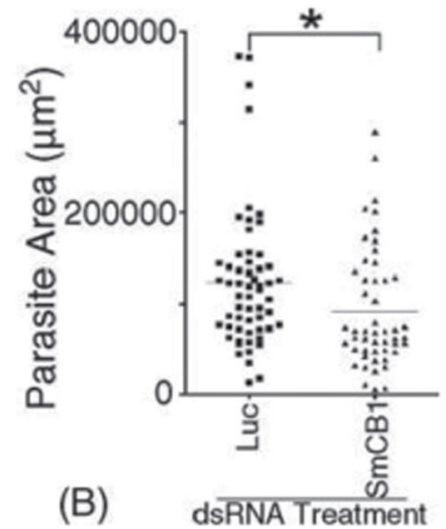

Figure 1.

In Schistosoma mansoni, RNAi induced by introduction of dsRNA at $3 \mathrm{~h}$ after mechanical transformation of schistosomules from cercariae has effects that persist for at least 3 weeks in vivo. (A) Examples of schistosomes recovered by portal vein perfusion 3 weeks following electroporation with either luciferase-dsRNA (left) or SmCB1-dsRNA (right) and intramuscular injection into mice. (B) Population quantitation of schistosome size 3 weeks following dsRNA treatment $(* p<0.005)$. Scale bar $=330 \mu \mathrm{m}$. (Correnti et al., 2005).

Reprinted from Mol Biochem Parasitol, 143, Correnti, J. M., Brindley, P. J. and Pearce, E. J., Long-term suppression of cathepsin B levels by RNA interference retards schistosome growth, 209-215, (2005), with permission from Elsevier. 


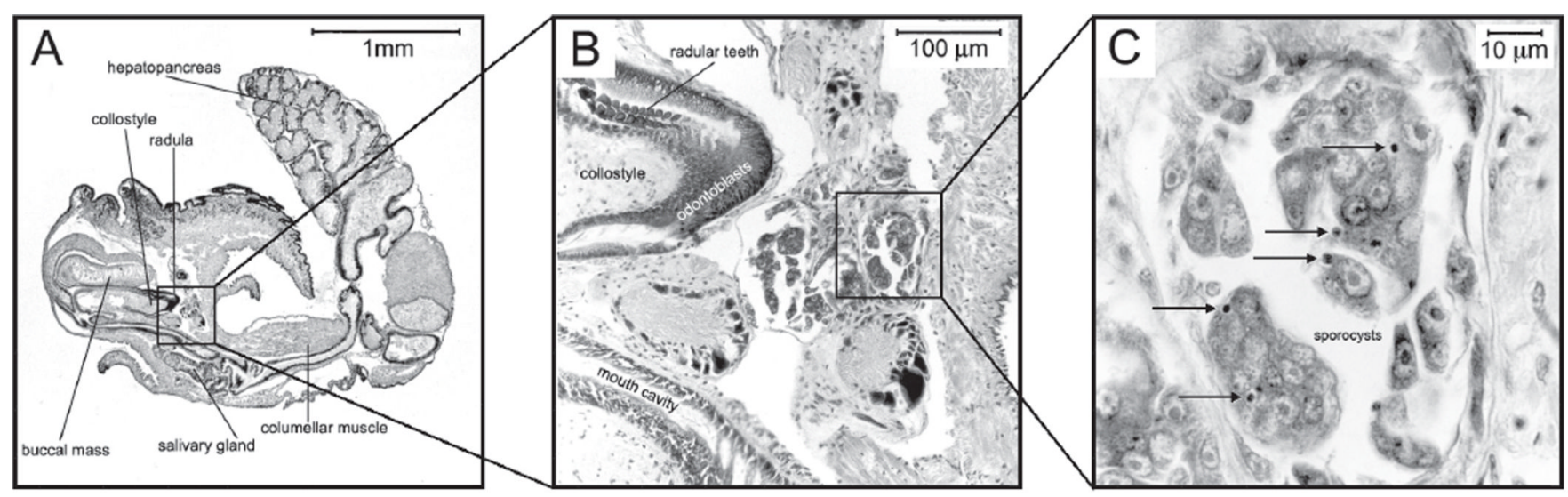

Figure 2.

Haematoxylin and eosin-stained paraffin section of Biomphalaria glabrata snail two weeks after infection with miracidia transformed by particle bombardment with gold microcarriers. Panel A: Overview section through the mouth, mid-body, head-foot and hepatopancreas/kidney region of the snail. The box outlines the kidney region that is infected with sporocyst. This boxed region is shown at higher magnifications in panels B and $\mathrm{C}$. The locations of other key anatomical features are indicated by text. Panel B: Higher magnification of snail tissues shown in panel A; the regions in and around the box outline include developing mother sporocysts. The radula of the snail is prominent in the upper left of the panel. The locations of other key anatomical features are indicated by text. Panel C: Higher power magnification of snail tissues from panel B, with a close-up image of sporocysts. The arrows show the position of gold particles in close proximity to the germ ball cells in the sporocysts. Scale bars are included at the top of each panel. (Heyers et al., 2003).

Reprinted from Exp Parasitol, 105, Heyers, O., Walduck, A. K., Brindley, P. J., Bleiss, W., Lucius, R., Dorbic, T., Wittig, B. and Kalinna, B. H., Schistosoma mansoni miracidia transformed by particle bombardment infect Biomphalaria glabrata snails and develop into transgenic sporocysts, 174-178, (2003), with permission from Elsevier. 

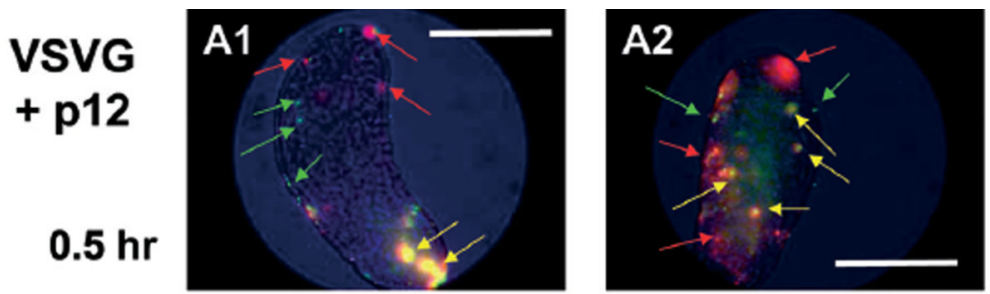

\section{VSVG}

$+$

p30

$1 \mathrm{hr}$
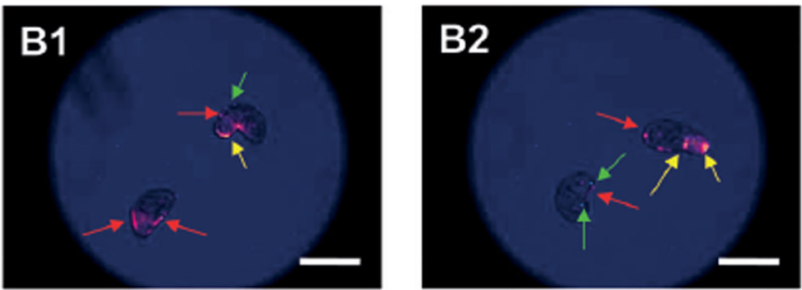

$0.5 \mathrm{hr}$
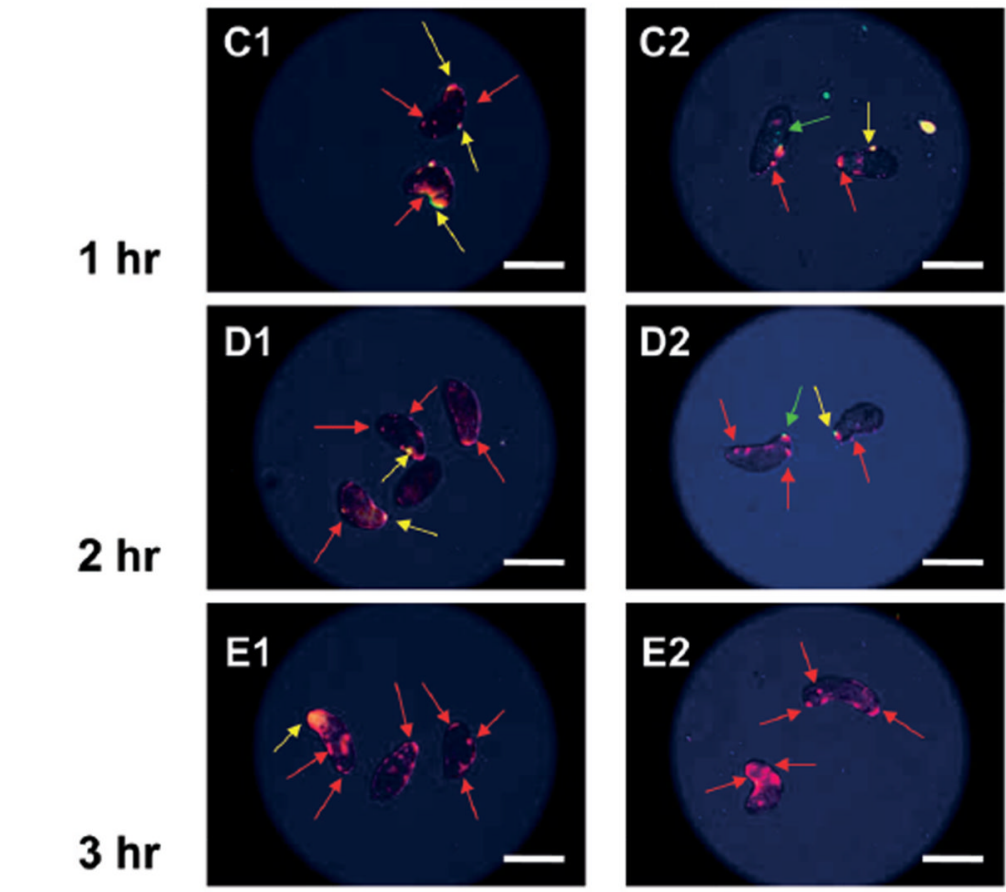

F1

F2

Control
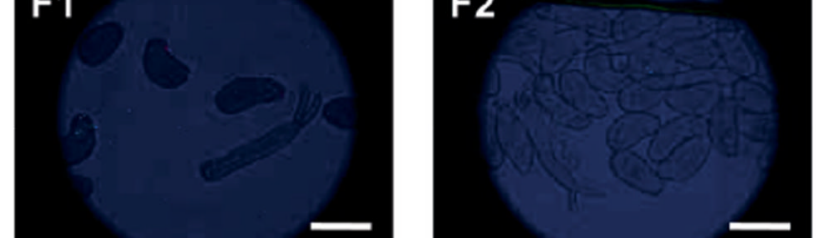

Figure 3.

Visualization of MMLV virions associating with the surface of schistosomules of Schistosoma mansoni. Schistosomula were exposed to supernatants of MMLV-VSVG (pLNHX-D70-EGFP) virions for $30 \mathrm{~min}$ to $3 \mathrm{~h}$, and then fixed and processed for dual immunofluorescence. Immunolabeling protocol involved anti-VSVG antibody with Alexa 488 goat anti-rabbit antibody (green signal, green arrows) and either anti-p12gag (A1-E1) or anti-p30gag (A2-E2) antisera with Alexa 594 donkey anti-goat antibody (red signal, red arrows). F1, F2, control schistosomules not exposed to virions but labeled with the antibodies. Images for green and red fluorescence were acquired separately and then overlaid (colocalization of red and green signals indicated by yellow signal, yellow arrows). 
Merged images of red, green, and bright field (blue) are presented. Bars: A1 and A2, 100 $\mu \mathrm{m}$; B1-F1, B2-F2, $50 \mu \mathrm{m}$ (Kines et al., 2006). Reprinted from Exp Parasitol, 112, Kines, K. J., Mann, V. H., Morales, M. E., Shelby, B. D., Kalinna, B. H., Gobert, G. N., Chirgwin, S. R. and Brindley, P. J., Transduction of Schistosoma mansoni by vesicular stomatitis virus glycoprotein-pseudotyped Moloney murine leukemia retrovirus, 209-220, (2006), with permission from Elsevier. 


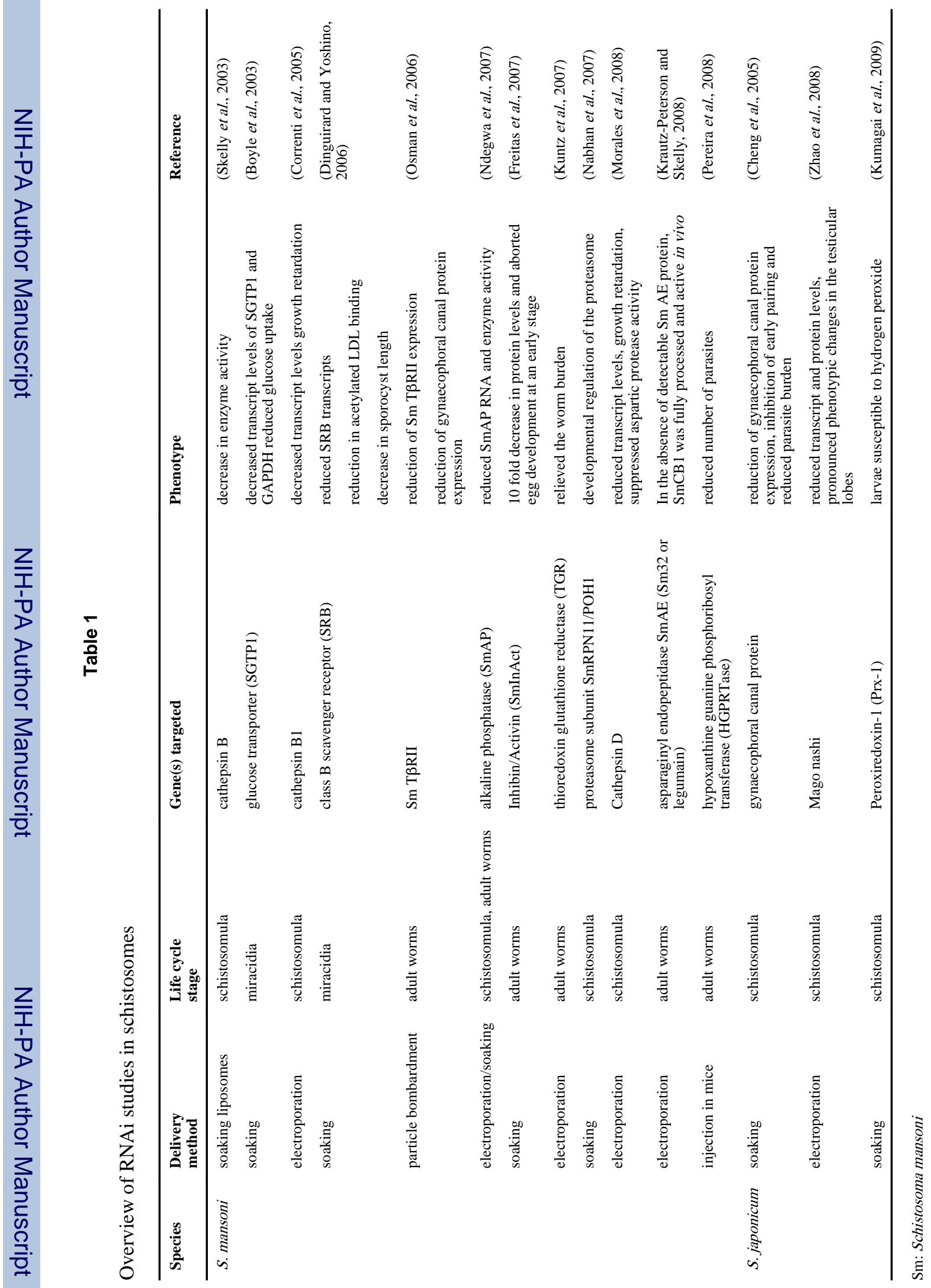




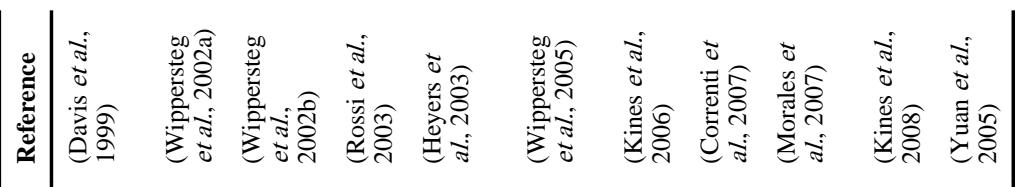

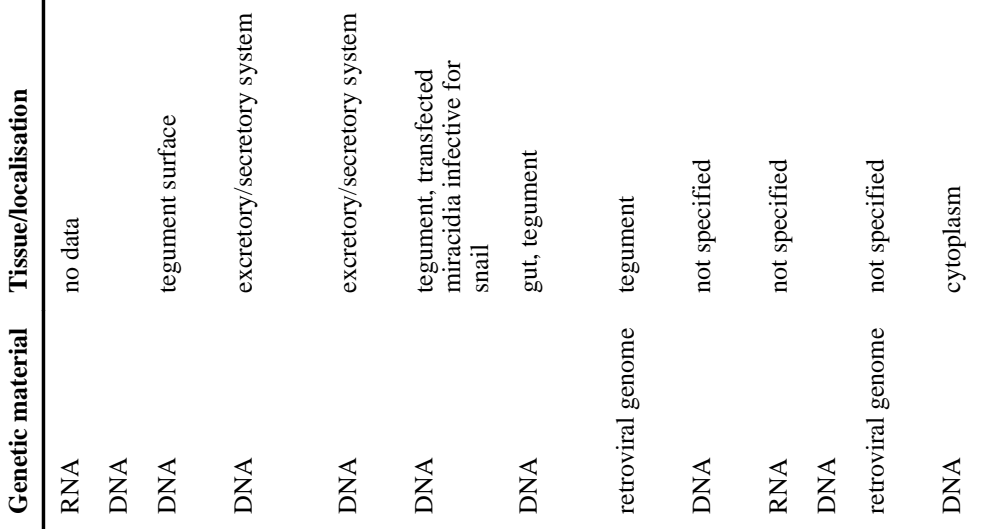

